Ana Patricia Melchor Organista 


\section{Motivación moral: enfoques empíricos y posibles vías para la investigación}

\section{Introducción}

En este texto se analiza la posibilidad de integrar los resultados de investigaciones empíricas a las principales discusiones teóricas en torno a la motivación moral, un territorio especialmente escabroso de la metaética, específicamente, de la psicología moral. Para esto se presentan, grosso modo, los dos principales debates en torno a la motivación moral, los argumentos e intuiciones de las que parten las distintas posturas respecto al origen y la naturaleza de la motivación moral.

Una vez establecido el panorama, se analizan dos casos de la psicología experimental, el Moral Dumbfounding de Jonathan Haidt (2014) y los casos de los psicópatas a los que acude Shaun Nichols (2004); ambos autores buscan aportar evidencia empírica en contra de una visión racionalista de la motivación moral. Se presentan los resultados de sus experimentos, así como la interpretación que sus autores les dan y se analiza qué tan exitoso resulta integrar dichos resultados al debate teórico, así como algunas consideraciones importantes para una metodología naturalista que nos permita generar propuestas teóricas que sean psicológicamente plausibles conforme a la evidencia empírica que tenemos.

\section{Los debates sobre el origen y naturaleza de la motivación moral}

Al hablar de motivación moral me referiré a aquello que nos lleva a actuar moralmente, ${ }^{1}$ otro tipo de definición estaría tomando de an-

1 Aquí sigo la tradición angloparlante, en la que la distinción ética/moral no es tan relevante como en otras discusiones. Considero que la ética es la reflexión 
temano alguna de las posturas de los debates que analizaremos. Sin embargo, es posible dar una definición más informativa e imparcial respecto a los debates; para ello, debemos partir de un grupo más amplio: el de la motivación normativa. En la motivación normativa se analizan, en general, los motivos que tenemos para actuar conforme a un conjunto de normas aplicables a una actividad; los motivos normativos pueden responder a sistemas normativos racionales o prudenciales, entre otros. Tengo una motivación del tipo normativa al seguir las reglas de tránsito o al guardar cierto código de etiqueta durante una cena formal, y de la misma manera, tengo una motivación normativa al seguir un conjunto de normas morales.

Ahora bien, la motivación moral es un tipo especial de motivación para la acción que resulta más problemática que otro tipo de motivaciones, especialmente porque los lineamientos de la moral no siempre se ajustan a los deseos de un individuo; en muchas ocasiones, la moral lleva al agente a realizar acciones contrarias a lo que podría significar su mayor placer o beneficio. Esta propiedad de ir en contra de lo que podría parecer la mejor opción, en sentido de beneficio para quien actúa, le da a la motivación moral un carácter distinto y mucho más complejo que la motivación para otra clase de acciones.

En resumen, la definición de motivación moral de la que partimos se puede formular como: "Aquellos elementos en nuestra psicología que nos llevan a actuar conforme a los lineamientos normativos distintivamente morales", y se enfoca en las razones que tiene el agente para actuar moralmente. Dicha explicación será válida para el agente que actúa y no necesariamente justificará la acción ante terceros. Es una explicación en el sentido de que las razones ofrecidas pueden ser malas, una justificación tendría que incluir buenas razones y ser aceptadas por terceros en tanto que son buenas.

Muchos podrán decir que nuestra motivación moral es exitosa en tanto que no vamos por allí mintiendo, robando o matando al resto de las personas, al menos la mayor parte de nosotros, la mayor parte del tiempo. De alguna forma somos capaces de seguir

sobre la moral y que la filosofía moral es, en ese sentido, un término intercambiable. Algunos llamarán "actuar conforme a la ética" a lo que yo llamo "actuar moralmente". 
ciertas reglas que nos permiten vivir en sociedad; sin embargo, no es claro por qué lo hacemos, cuáles son los procesos que nos llevan a ello. Por algún tiempo la motivación moral se dio por supuesta o al menos se creyó que no era tan problemática como lo es hoy en día, pero desde las aportaciones de Hume al tema y las reacciones de Kant y el grupo antihumeano hacia dichas aportaciones, los debates se han vuelto más intrincados y la división entre posturas es bastante pronunciada.

En la discusión actual hay dos elementos principales que se discuten cuando se analiza la motivación moral desde la filosofía y es que los puntos que se sostienen acerca de ella parecen ser incompatibles; David O. Brink ${ }^{2}$ nos presenta las ideas que son comúnmente compartidas respecto a la motivación moral y las divide en cuatro puntos:

1. Los juicios morales expresan creencias.

2. Los juicios morales acarrean motivación.

3. La motivación involucra un deseo o pro-attitude. ${ }^{3}$

4. No hay una conexión necesaria entre cualquier creencia y cualquier deseo o pro-attitude.

Los primeros dos puntos hacen referencia al debate internalismo-externalismo sobre el juicio moral, y los últimos dos al debate sobre la teoría humeana de la motivación.

El debate humeanismo-antihumeanismo sobre la motivación moral busca definir de dónde viene la motivación: si de nuestra racionalidad, en forma de creencias, o de nuestras pasiones, en forma de deseos; el debate sobre el internalismo o externalismo acerca del juicio moral busca establecer si hacer un juicio moral nos lleva a estar motivados o no: si formular un juicio moral respecto a

2 Brink, David O., (Oct. 1997), “Moral Motivation”, Ethics, Vol. 108, No.1, pp. 4-32, p. 6.

3 Este concepto es introducido por Donald Davidson en su teoría de la acción que, a grandes rasgos, sostiene que para que un agente actúe debe tener una razón y esa razón se traduce en una "actitud favorable" hacia dicha acción, dicha actitud se compone de un deseo y una creencia, tal como la reformulación que hace Michael Smith sobre Hume más adelante. Una razón puede socavar a otra, aun cuando exista el autoengaño y el agente piense que su razón para actuar fue $p$ cuando en realidad fue $q$. 
algo nos impulsa ya a actuar en consecuencia y nos impulsa necesariamente, aunque el impulso puede ser intenso o casi imperceptible, o si formular un juicio moral no compromete en ningún sentido nuestra motivación. El primero, entonces, se entiende como el problema de adjudicarle el origen de nuestra motivación moral a la razón a la pasión y el segundo como el problema de si hacer un juicio moral nos motiva necesariamente a actuar acorde a él o no; por supuesto, existen versiones fuertes y versiones moderadas de ambas partes de las tesis.

Hume defiende que, puesto que la moral se dirige a nuestras acciones, los elementos cognitivos quedan fuera de la ecuación en tanto que no pueden llevar a acciones, no pueden mover la voluntad. Esto se justifica en que una conclusión de la razón es la asignación de los criterios de verdad o falsedad, a los que se llega por medio de un acuerdo general, y puede referirse o a una relación real de ideas o a la relación entre existencia real y una cuestión de hecho. Las cuestiones de hecho son inferidas por la razón y cuando se alcanzan se consigue de ellas un "frío asentimiento de la razón", la razón reconoce que es verdad, pero no tiene otra reacción que ese reconocimiento cognitivo, pero dicho reconocimiento no lleva al agente a ningún estado conativo, necesario para la acción y ya que el papel de la moral es regular nuestras vidas y nuestras acciones, los estados cognitivos no tienen papel en dicha regulación.

Hume nos describe a las pasiones como hechos y realidades originales y completas en sí mismas, son o una existencia original o una modificación de la existencia y se generan a sí mismas. No representan cómo son las cosas, no tienen contenido proposicional, nada las causa, mucho menos estados cognitivos. Aquí las pasiones se asemejan bastante a elementos a priori, pues no requieren de nada del mundo y surgen de nosotros completas y nos llevan a la acción.

Para muchos la propuesta de Hume desemboca en un subjetivismo y en un emotivismo. Pues, aunque Hume sostiene que los mismos objetos tendrían que generar las mismas reacciones de atracción o repulsión a los hombres, en tanto que comparten una misma naturaleza, en la práctica podemos observar que las emociones de las personas, lo que les resulta atractivo o repulsivo, varía casi de cabeza en cabeza y muchos incluso piensan que un consenso en términos de inclinaciones personales es imposible.

Hume sostuvo que los juicios morales dependen de estados psicológicos e individuales del agente que los formula y que el con54 
tenido de esos juicios morales no es más que sus deseos, más que las pasiones que ciertos objetos le producen. Y para que esta postura se sostenga, se afirma que esos deseos pueden ser inconscientes o no accesibles al sujeto que los experimenta. Este último punto va en contra de las intuiciones de la mayoría, pues cuando nos comprometemos con algo solemos defender que tenemos razones e intenciones conscientes para ello, o podemos ofrecerlas una vez que hemos realizado la acción.

Además, una propuesta humeana no explica por qué la intuición general nos dice que los argumentos morales provienen de la razón y por qué resultan tan importantes. Si se acepta toda la propuesta humeana, se renuncia a la idea de un sistema de normas objetivas (o al menos que aspire a serlo) y a la idea de que podemos modificar nuestras normas y conductas morales conforme a la razón.

Los experimentos que se presentan a continuación se concentran en aportar evidencia empírica para una visión humeana de la motivación moral, pero, sobre todo, buscan mostrar que una concepción racionalista sobre la motivación moral no es viable según dicha evidencia.

\section{Evidencia empírica contra el racionalismo y algunos reparos}

Los debates y argumentos de cada parte que hemos presentado arriba caen en un círculo vicioso de apelación a intuiciones y contraejemplos para esas intuiciones, y si bien es cierto que es así como suele proceder una argumentación filosófica, en el caso de la motivación moral, el debate puramente conceptual da la sensación de no dirigirse hacia ningún lado. De allí surge la necesidad de analizar estudios empíricos relacionados con los elementos que se debaten, que buscan aportar información valiosa tanto sobre la forma en que generamos nuestros juicios morales como sobre la relación que tienen esos juicios con nuestras acciones.

\section{Moral dumbfoundingः Jonathan Haidt}

Haidt, en su libro The Happyness Hypothesis, retoma las analogías que buscan explicar las divisiones de la mente que se han ofrecido a lo largo de la historia y hace referencia a la conocida metáfora 
utilizada por Platón en el diálogo Fedro: la analogía de una carroza llevada por dos caballos, que representan los deseos y pasiones humanas, el primero odioso a la vista y el segundo como un hermoso corcel; este último representa las pasiones elevadas, como el deseo por el honor, mientras el otro representa las bajas, como la búsqueda de placeres corporales, los apetitos, y un jinete simboliza a la razón, quien puede guiar ambos caballos y elevarse poco a poco hasta llegar al mundo de las ideas. Sin embargo, esta analogía dista bastante de nuestra realidad: no todo lo que hacemos va guiado ordenadamente por la razón, por más atractiva que pueda parecernos la idea. Haidt propone una nueva analogía, en la que cambia la carroza y los caballos por un enorme elefante, sobre el que nuestro jinete tiene poco control y al que sólo puede acceder parcialmente. Aquí la razón sigue siendo representada por el jinete, pero resulta ser un guía más limitado de lo que se creyó en un principio. Valerie Tiberius la describe así:

Nuestro ser no-reflexivo es como un enorme y decidido elefante, $y$ nuestro ser reflexivo es el pequeño jinete montado encima, con un control más bien limitado. Nuestro ser reflexivo no es tan inteligente ni tan poderoso como lo requiere la idea del control racional. ${ }^{4}$

Distintos experimentos psicológicos parecen apoyar las afirmaciones del sentimentalismo, si bien en ellos se mezclan los elementos motivacionales que vimos en los apartados anteriores, especialmente el papel que tienen deseos y creencias en nuestras acciones morales. En el campo de la psicología moral existe una contienda entre los partidarios de la llamada "Affective-primacy theory" y la "Cognitive primacy theory", que recuerda a la pregunta de qué es primero, si el huevo o la gallina. Esta contienda busca distinguir qué elemento se procesa primero en las reacciones de los individuos ante los objetos, si elementos cognitivos y no afectivos, como el reconocimiento y clasificación de un objeto, o elementos afectivos, como la reacción emocional que le produce ese objeto, aunque no se haya reconocido o clasificado previamente. ${ }^{5}$

4 Tiberius, Valerie, The reflective life, living wisely with our limits, Oxford University Press, N.Y., 2008, p. 18.

5 Esta discusión puede darnos luz sobre la discusión de la motivación moral, si bien es importante notar que se trata de las primeras reacciones inmedia- 
A favor del sentimentalismo, tenemos los experimentos de Jonathan Haidt y su equipo, presentados en el artículo The affective dog and its rational tale: Intuition and attunement. ${ }^{6}$ En él se busca demostrar que, en muchas ocasiones, los agentes no pueden dar razones que justifiquen o respalden sus juicios morales. A este fenómeno, de no poder ofrecer razones para un juicio moral pero continuar sosteniéndolo dogmáticamente, se le conoce como Moral Dumbfounding y muchos de los defensores de este fenómeno afirman que todos nuestros razonamientos morales tienen un proceso similar detrás. Sostienen que el dumbfounding tiene errores en la asignación de razones post hoc a los sentimientos y es por eso que llama nuestra atención.

Defienden, además, que el papel de la razón en las justificaciones morales es mínimo o inexistente, pues la razón entra en acción sólo después de que los sentimientos y las intuiciones actúan en nosotros. La razón tiene el papel de un abogado, reconstruye el caso, buscando darle un sentido que parezca más sólido que la simple intuición o el sentimiento, y lo que identificamos como un juicio moral, al que le atribuimos el carácter de racional, es en realidad esa explicación creada después de que otros estados mentales originaran el juicio.

Sin embargo, Jacobson se opone a esta propuesta y traza una línea de defensa importante en contra de esta visión de la razón como reconstructora, confabulando razones morales post factum para los juicios que se emiten; si bien no se opone a darle un papel protagonista a los sentimientos, a las respuestas afectivas, sí va en contra del rol que se le asigna a la razón:

De este modo, mientras acepto una forma moderada de la tesis de la "primacía del afecto" de acuerdo a la cual factores que ignoramos juegan un papel significativo en dirigir los juicios evaluativos, las posibles versiones de esta tesis no pueden ser tan fuer-

tas del agente y no como tal de su predisposición a actuar. Las conclusiones apuntan a que el contexto en el que se encuentra la persona determina qué elementos surgen primero, si juicios afectivos o juicios cognitivos, indicando que ninguna de las dos posturas es correcta, no es verdad que siempre lo primero es un juicio afectivo ni es verdad que lo primero sea un juicio cognitivo.

6 (Jul.2014),"The affective dog and its rational tale: Intuition and attunement", Ethics Vol. 124, No. 4, pp. 813-859. 
tes como para implicar la (casi) impotencia causal de las razones y el razonamiento, tal como lo afirma la postura pesimista. ${ }^{7}$

Esto en tanto que de que un agente no pueda ofrecer razones para su juicio no se sigue que no existan razones válidas detrás de él. Es posible, por ejemplo, que el juicio haya sido hecho por otras personas en el pasado, por medio de la observación y la racionalidad práctica; que luego se convirtiera en una norma moral y pasara al agente del experimento por tradición. El hecho de que obtuviera el juicio por tradición y no lo realizara por sí mismo, no implica que no exista un razonamiento de fondo que lo justifique.

Razones pueden ser ofrecidas y esas razones pueden estar basadas en un análisis profundo sobre las consecuencias e implicaciones de una acción específica. Incluso sostiene que preferir no hacer algo porque resulta desagradable cuenta como un argumento en contra de la acción, pues existen razones detrás de eso, aunque no se puedan ofrecer todas en el momento en que se cuestiona.

Es importante tener en cuenta aquí que nuestras creencias sobre el mundo y las normas sociales que interiorizamos no son sólo producto de nuestras observaciones y reflexiones individuales, sino que vamos aprendiendo del trabajo y el análisis de otros, y en el caso específico que estamos tratando, la educación moral que hemos recibido pude producir en nosotros deseos y disposiciones de larga duración que no necesariamente se han llevado al análisis y esto no implica que sean irracionales o viscerales. El reto, una vez más, consiste en someterlos al análisis y encontrar si se puede ofrecer una explicación racional de ellos. ${ }^{8}$

7 Jacobson, D., "Moral Dumbfounding and Moral Stupefaction," en M. Timmons (Comp.) Oxford Studies in Normative Ethics: Vol. 2, Oxford University Press, U. K., 2012, p. 293.

8 Quisiera aquí hacer mención de otro de los experimentos que Haidt emplea para apoyar su propuesta, evidentemente humeana. La serie de experimentos son dirigidos por Eric Schwitzgebel y Joshua Rust y buscan investigar si los eticistas, aquellos que se dedican a la reflexión ética, muestran cambios significativos en su comportamiento, se analizan grupos de profesores de ética y profesores de otras ramas en conferencias, para observar si existen cambios en parámetros tales como la intensidad de las voces que se escuchaban mientras el conferencista hablaba, la cantidad de basura que dejaban en la sala y el número de azotes al abrir y cerrar la puerta; otro de los parámetros 
En general, las acciones que son tachadas de incorrectas o de desagradables, para las que parece que los agentes no cuentan con una explicación satisfactoria, tienen de fondo razones por las que se consideran incorrectas o desagradables, y a menudo esas razones se relacionan con las relaciones sociales o con la supervivencia de la especie.

\section{Los perfectos amoralistas, Shaun Nichols}

En el tercer capítulo de su libro del 2004, Is it Irrational to be Amoral? How Psychopaths Threaten Moral Rationalism, ${ }^{9}$ Shaun Nichols busca demostrar, por medio de experimentos mentales sobre psicópatas y experimentos reales en psicópatas, que la propuesta racionalista es inverosímil. Su estrategia consiste en identificar dos tipos de teorías racionalistas: las que apelan a los elementos conceptuales y las que lo hacen a elementos empíricos. Los argumentos conceptuales apelan a que es una verdad conceptual que un requerimiento moral es una razón para la acción y que hablar de razones implica la racionalidad; los argumentos empíricos sostienen que nuestros juicios morales se derivan de nuestras facultades o capacidades racionales. Los argumentos conceptuales defienden

fue el robo en los libros de las bibliotecas de las instituciones. Las diferencias no fueron significativas, y en el caso de los libros de la biblioteca, los profesores de ética tuvieron estándares de robo más altos (a excepción de la basura dejada en las conferencias de ética ambiental, en donde claramente había una diferencia). La intuición que subyace al estudio y de la que hace uso Haidt es que si la deliberación puede modificar nuestro comportamiento, entonces personas que dedican tanto tiempo a deliberar sobre asuntos éticos, necesariamente tendrían que presentar un cambio en su comportamiento, pero en tanto que no hay tal cambio, Haidt piensa que podemos usarlo como prueba de que la razón no modifica nuestro comportamiento. Los mismos desarrolladores del experimento escriben que sus resultados pueden leerse de otras formas, que no apoyan la propuesta humeana sobre la racionalista. (vid.: Rust, Joshua y Schwitzgebel, Eric, "The Moral Behavior of Ethicists and the power of reason", en H. Sakissian y J. Cole Wright (eds.), Advances in Experimental Moral Psychology, Bloomsbury Publishing, London, 2014).

9 Nichols, Shaun, Sentimental Rules: On the Natural Foundations of Moral Judgment, Oxford University Press, U. K., 2004. 
que la racionalidad es parte de nuestro concepto de moralidad y los empíricos apelan a hechos de nuestra psicología. De esta forma, nuestros conceptos que tienden a la racionalidad pueden estar equivocados y es posible que, aun así, nuestra psicología implique elementos racionales. Sin embargo, Nichols busca demostrar que ambas defensas están equivocadas.

El experimento que usa para probar que el racionalismo conceptual está equivocado se relaciona directamente con el debate entre internalismo y externalismo sobre el juicio moral, pues el caso del amoralista, que es capaz de formular un juicio moral sin tener motivación, presenta una fuerte paradoja para el internalista, que, recordemos, defiende que al formular un juicio moral sincero, el agente que lo formula debe ya estar motivado a actuar de acuerdo con él. La estrategia del internalista, entonces, es decir que el juicio formulado por el amoralista no es sincero, es un juicio entrecomillado, el amoralista no expresa que robar está mal, sino que afirma "robar está mal". ${ }^{10}$ Teniendo en mente esta estrategia, Nichols preguntó a distintos estudiantes que no estaban familiarizados con los debates, si los juicios morales formulados por los psicópatas eran genuinos. Las intuiciones de la gran mayoría señalaron que sí, que el psicópata puede hacer juicios morales genuinos, pero simplemente el contenido de ese juicio no le importa. Para Nichols, ésta es una muestra de que nuestro concepto está equivocado, que la formulación, supuestamente racional, de los juicios morales no conlleva motivación.

Para mostrar que el racionalismo empírico está también equivocado, acude a estudios que buscan describir qué es lo que falla en la psicología de los psicópatas, si la parte afectiva o la parte cognitiva - esto se relaciona directamente con el debate sobre el humeanismo o antihumeanismo en la motivación moral. Nichols señala que las teorías que defienden que la falla en los psicópatas es una falla racional, no pueden mostrar cómo ésa se limita sólo a los casos de los amoralistas y no se presenta en otros casos. ${ }^{11} \mathrm{Su}$

10 La estrategia es mucho más compleja, esta versión es en exceso simplificada; la idea principal es que el amoralista sólo repite lo que otros juzgan sobre algo, sin creerlo él mismo. De esta forma, los Internalistas sobre el Juicio Moral pueden defender que su juicio no es sincero y es por esto que no hay motivación.

11 Específicamente se centra en la incapacidad de los psicópatas para distinguir entre lo que es moralmente incorrecto y lo que es convencionalmente inco- 
estrategia consiste en señalar que estas propuestas no son exitosas y que la respuesta a qué es lo que anda mal con los psicópatas se inclina más a fallas en sus capacidades afectivas, como la de los psicópatas para reconocer señales de angustia en las expresiones de las personas, lo que, en agentes afectivamente normales, activa un mecanismo inhibidor de violencia.

Recurre a estudios que señalan que el origen de la incapacidad de los psicópatas para hacer juicios morales genuinos es su incapacidad de experimentar simpatía, culpa, remordimiento y empatía, todas respuestas afectivas. Concluye, pues, que la sensibilidad emocional juega un papel clave en el juicio moral y que tendríamos que inclinarnos por una explicación sentimentalista (y humeana) de la moralidad.

Jeannette Kennett responde puntualmente a las conclusiones de Nichols y defiende que el racionalista puede dar una explicación de fallas cognitivas exclusivas para el psicópata y que los elementos empíricos a los que hace referencia son de hecho compatibles con visiones racionalistas.

Kennett sostiene que existen fallas cognitivas que distinguen a los psicópatas y pueden explicar sus problemas al distinguir principios morales y al seguirlos. Para ello, cita los estudios de Robert Hare sobre los psicópatas, en los que se concluye que los psicópatas tienen una especie de desorden o discontinuidad al momento de seguir sus metas, lo que les impide ser competentes al deliberar o tener autocontrol.

Luego pasa a estudios psicológicos que muestran que el autocontrol ${ }^{12}$ es fundamental para el comportamiento ético. Estos estudios muestran que conforme nos desarrollamos, vamos generando la capacidad de lidiar con los estímulos externos, lo que nos

rrecto; la capacidad de distinguir entre estos dos juicios es un paso esencial en el desarrollo moral. Una de las explicaciones de los racionalistas es que los psicópatas no son capaces de ponerse en el lugar del otro y eso les impide hacer la distinción, pero los niños autistas son capaces de distinguir entre principios morales y principios convencionales y tampoco son capaces de generar empatía por otros; Nichols explora distintas propuestas que fallan al asignar un problema cognitivo a los psicópatas, por ello, concluye que lo que anda mal en los psicópatas no es una falla en sus capacidades racionales.

$12 \mathrm{El}$ autocontrol es entendido como un proceso controlado por medio del cual los agentes alteran sus acciones en pos de una meta. 
permite continuar con nuestras metas, aun frente a distintos estímulos distractores. ${ }^{13}$

Kennett concluye que la única forma que tendrían los partidarios del sentimentalismo para eliminar la propuesta racionalista sería mostrar que los agentes son incapaces de juzgar moralmente y de actuar según la visión racionalista, pero no hay suficiente evidencia para probar algo así.

Por su parte, Besser-Jones ${ }^{14}$ sostiene que existen investigaciones empíricas que muestran que la razón práctica puede jugar un papel principal tanto en la formulación de juicios morales como en la motivación moral. Ella apela también a los estudios sobre el autocontrol y los procesos deliberativos; a la forma en que regulamos nuestro comportamiento.

El papel de la razón aquí puede ser el de evaluar los propios deseos y reflexionar acerca de las posibles razones que se tienen para perseguir cualquiera de esos deseos; esto se relaciona con nuestras ideas de racionalidad práctica, la reflexión sobre aquello que se debería hacer, una concepción de deseo muy similar a los deseos motivados de Nagel.

Los experimentos acerca de la deliberación y elección de metas son complejos, en tanto que no se tiene acceso directo ni a la deliberación ni a las metas genuinas que los agentes tienen. Sin embargo, parece evidente que procesos como éstos requieren de funciones cognitivas propias de la corteza frontal, y la evidencia empírica muestra que agentes con daños en la corteza frontal ${ }^{15}$ tienen serios problemas al tomar decisiones y controlar su comportamiento frente a deseos fuertes.

Además, importantes teorías psicológicas ${ }^{16}$ sobre el proceso de autorregulación recurren a razonamientos deliberativos conscientes: una función, "the assessment function", evalúa críticamente las posibles metas y medios para decidir cuál perseguir y otra función, "the locomotion function", nos lleva a la acción; ambas funciones

13 Esto se concluye de los experimentos de Kochanska y Aksan (2004) y Walter Mischel (1981).

14 Besser-Jones, Lorraine, (Abr. 2012), "The role of practical reason in an Empirically Informed Moral Theory", Ethical Theory and Moral Practice, Vol. 15, No. 2, pp. 203-220.

15 Específicamente en la corteza frontal ventromedial; Banfielt et al. (2004).

16 Kruglanski and colleagues (2000). 
se refieren principalmente a aquellos deseos que el agente considera como mejores y no a los deseos más fuertes, que dejarían a la razón como un simple instrumento de obtención de deseos previos y que no pasan por un proceso de deliberación.

He argumentado que las metas morales son vistas más plausiblemente como el producto de la reflexión racional en consideraciones normativas relevantes, la aceptación del agente de esa reflexión transforma ciertos deseos y fines en metas; las metas morales son entonces deseos privilegiados o valorados. ${ }^{17}$

Besser-Jones concluye que tenemos suficientes bases empíricas para asignarle un rol principal a la razón práctica tanto en la formación de nuestros juicios morales como en nuestra motivación moral.

\section{Integrar la evidencia a los debates}

En los experimentos llevados a cabo por Haidt y Nichols parece evidente que, como todos los experimentos, se parte de nociones e intuiciones previas de los autores que, en este caso, favorecen una visión humeana sobre la motivación moral y buscan socavar la propuesta racionalista, o al menos decir que la evidencia empírica apunta al lado contrario. Sin embargo, los resultados de ambas investigaciones pueden leerse y encajar con distintas teorías, incluso algunas de corte racionalista.

Cualquier propuesta que busque seguir una metodología naturalista está comprometida a analizar la información empírica disponible y buscar complementar y justificar o contrastar y modificar su propuesta teórica. Es necesario ser cuidadosos al momento de elegir la información empírica a utilizar y comprobar que las pruebas realizadas apoyen las hipótesis que se presentan, así como que la información proporcionada sea relevante e, incluso, si hay formas alternas de interpretar los resultados obtenidos. Jeanette Kennett ilustra acertadamente los principales problemas que enfrentamos al integrar las investigaciones empíricas a debates conceptuales complejos, al expresar su preocupación por que:

17 Besser-Jones, op. cit., p. 216. 
[... filósofos (y otros) puedan tender a extrapolar más allá de la información y tracen conclusiones filosóficas que la información no apoya. [...][La primera razón para esto es que] la información que se cita puede no constituir toda la información relevante y puede que los filósofos no estén en condiciones de evaluar su calidad. [...] $[$ Su segunda razón es que] se debe tener especial cuidado al aplicar descubrimientos empíricos a cuestiones filosóficas del razonamiento moral, pues estas consideraciones no son meramente descriptivas. ${ }^{18}$

Quisiera concentrarme justamente en el segundo punto, y es que las reflexiones morales buscan establecer parámetros y restricciones normativas y conceptuales. Kennett postula que, en tanto que es así, es posible que la evidencia empírica no afecte los debates que hemos venido tratando; sin embargo, considero que la evidencia empírica debe afectar a los debates, pues debemos partir de cómo es que funcionamos para generar sistemas normativos y conceptos morales que puedan ajustarse a los conocimientos que nos aportan los elementos descriptivos. Nuevamente, tendríamos que buscar que nuestras teorías sobre la moralidad sean psicológicamente plausibles.

La filosofía se distingue de las ciencias en tanto que no se queda en un análisis descriptivo y, especialmente en moral, se busca establecer también criterios normativos. Integrar elementos descriptivos no significa dejar de lado el aspecto normativo de la filosofía moral, que busca no sólo presentar cómo tendríamos que actuar, sino que hay casos en los que aspira incluso a brindar parámetros para mejorar nuestras prácticas morales. El integrar elementos descriptivos, pues, buscará que las teorías formuladas no contradigan o ignoren aspectos fundamentales de nuestra psicología.

Considero que la evidencia presentada no nos da pruebas contundentes para decidirnos por alguna postura en los debates sobre la motivación moral, y que debemos tener cuidado al citar evidencia empírica. Sin embargo, dicha evidencia nos muestra principalmente dos cosas: que los elementos conativos juegan un papel muy importante en nuestra moralidad -si bien esto no elimina una posible explicación racionalista- y que la mayoría de nuestros procesos mentales son automáticos, pero la moral tendría

18 Kennett, Jeanette (Ago. 2006), "Do psychopaths really threaten moral rationalism?”, Philosophical Explorations, Vol. 9, No.1, pp. 69-82, p. 69. 
que formar parte de nuestros escasos procesos controlados para corresponderse con las intuiciones que le atribuimos.

Este último punto es compatible con la propuesta de los deseos motivados de Thomas Nagel, deseos que tienen un proceso de deliberación previo; un estudio empírico más profundo sobre la deliberación y el autocontrol podría ayudarnos a fundamentar esta propuesta. Incluso me inclino a afirmar, junto con otros optimistas acerca de la moral, que podríamos hacer de estos procesos deliberativos un hábito, de forma que pasen a ser parte de las respuestas automáticas de nuestro comportamiento. Por el momento, basta con notar que los deseos motivados respetan el papel fundamental que tienen los deseos en nuestro comportamiento y que la evidencia apunta a que un proceso deliberativo que guíe esos deseos es posible en un nivel importante. Esto se corresponde con la fuerte intuición general de la posibilidad de perfeccionamiento moral, pues, tal como lo describe Besser-Jones: "La moralidad funciona en la presuposición de que tenemos control sobre nuestras acciones; que podemos deliberar y reflexionar acerca de qué hacer; que podemos esforzarnos para mejorar nuestro comportamiento". ${ }^{19}$

\section{Conclusiones}

Estamos aún lejos de encontrar una respuesta definitiva para las preguntas acerca del origen, los elementos y mecanismos que conforman nuestra motivación moral y, al discutir al respecto, debemos tener presente que la postura que se tome en estos debates influirá considerablemente en todas las propuestas subsecuentes en ética normativa y aplicada.

En cualquier teoría que formulemos, será importante tener presente que el control que tenemos es limitado y que necesitamos continuar investigando las partes de nuestro elefante que nos son más ajenas; así como ser conscientes de que el proceso de mejorar nuestras prácticas morales no será sencillo, que somos seres altamente complejos y que a nuestras teorías seguramente siempre se les escaparán elementos importantes. Pero todas estas precauciones no eliminan la posibilidad de una visión racionalista, específcamente, de una versión detallada de racionalidad práctica.

19 Besser-Jones, L., op. cit., p. 209. 